\title{
Using Micro-Beam Techniques to Infer Meteorite Abundances of the Jurassic
}

\author{
C. E. Caplan ${ }^{1,2}$, G. R. Huss², H. A. Ishii ${ }^{2}$, J. P. Bradley², P. Eschbach³ , B. Schmitz ${ }^{4,2}$ and K. Nagashima² \\ 1. Department of Geology and Geophysics, University of Hawai'i at Mānoa, Honolulu, HI. \\ 2. Hawai'i Institute of Geophysics and Planetology, University of Hawai'i at Mānoa, Honolulu, HI. \\ 3. Electron Microscopy Facility, Oregon State University, Corvallis, OR. \\ 4. Department of Physics, University of Lund, Lund, Sweden.
}

We have recently begun a major collaborative project to determine the relative abundances of meteorite types over geologic time using remnant extraterrestrial chrome-spinels. These grains resist weathering and are preserved in terrestrial limestone [1]. The assignment of chrome-spinels to meteorite types requires multiple tools and techniques. The combination of chemical composition, oxygen isotopes, and the mineralogy and chemical composition of inclusions within the chrome-spinel define the host meteorite type. This work concentrates on samples from the Jurassic ( $160 \mathrm{Myr}$ ago).

Element abundances provide a first order classification of the grains. Abundances were determined by wavelength-dispersive (WDS) analysis on the JEOL JXA-8500F field emission electron microprobe at the University of Hawai'i (UH) using an accelerating voltage of $20 \mathrm{keV}$, a beam current of $20 \mathrm{nA}$, and varying beam diameters $(1-10 \mu \mathrm{m})$. Chrome-spinel compositions vary among meteorite types and can be used to classify the parent meteorites. In this study, grains were considered as classified if they matched 5 out of 8 elements $(\mathrm{Cr}, \mathrm{Fe}, \mathrm{Al}, \mathrm{Ti}, \mathrm{Mg}, \mathrm{V}, \mathrm{Mn}, \mathrm{Zn}$ ) to a chrome-spinel from a known meteorite type. Some grains were not classifiable using this method alone.

Oxygen isotopes are also an important way to distinguish between host meteorite types, as well as between terrestrial and extraterrestrial samples. The Cameca ims 1280 ion microprobe (SIMS) at UH was used to measure oxygen-isotopes (see [2] for details). Measurement pits were examined using a JEOL 5900 LV scanning electron microscope (SEM), and data from pits that intersected cracks or secondary alteration were eliminated from the data set. Figure 1a shows a combination of element and oxygen-isotope abundances, where oxygen is reported as $\Delta^{17} \mathrm{O}$ (indicated by color). In oxygen-isotope space, $\Delta^{17} \mathrm{O}$ is the distance away from the terrestrial fractionation line $\left(\Delta^{17} \mathrm{O}=\delta^{17} \mathrm{O}-0.52 \times \delta^{18} \mathrm{O}\right)$, where all terrestrial samples plot $\left(\Delta^{17} \mathrm{O}=0\right)$. The combination of element abundances and oxygen isotopes shows a clear distinction between chrome-spinels from ordinary chondrites (orange-red) and those from other types of meteorites.

Even with the combination of element and oxygen-isotope compositions, classification of some grains remains ambiguous. Inclusions within the extraterrestrial chrome-spinels provide additional information that may improve grain classification [3]. These inclusions are too small $(2-4 \mu \mathrm{m})$ to collect reliable compositions using the electron microprobe due to the inclusion of signal from the surrounding chromespinel. Fortunately, sections of the inclusions can be extracted using a focused ion beam (FIB) instrument and analyzed using the scanning transmission electron microscope (STEM). Using the UH FEI Helios 660 dual beam FIB-SEM at $15 \mathrm{kV}$, energy-dispersive (EDS) analyses of major- and minor-element abundances were measured to identify inclusions. FIB sections of inclusions were extracted and the polished at $5 \mathrm{kV}$ to reduce surface damage. We used the UH FEI 80-300 kV Titan dual Cs-corrected and monochromated STEM to image and analyze element chemistry at $300 \mathrm{kV}$. 
Two inclusion-bearing grains illustrate the potential of using inclusions to help with identification of the parent meteorite types. Inclusions from both grains are more complex than expected. Grain 1 contains amorphous inclusions with pyroxene-like compositions that are surrounded by layers of iron oxide and silica glass, which probably formed during terrestrial alteration. These inclusions are too heavily altered to be used to classify this grain. The inclusions in grain 2 are in close proximity and both are pyroxenes, but their compositions suggest that the two inclusions came from different meteorite types (an implausible result). Detailed element maps were collected at $200 \mathrm{kV}$ using the Oregon State University 80-200 kV Titan ChemiSTEM with four EDS quadrant detectors to better understand these inclusions (Fig. 1b). Both inclusions exhibit a thin layer of iron oxide at the chrome-spinel boundary, most likely due to terrestrial alteration. Inclusion 2 has a more-pronounced rim and a less iron-rich pyroxene composition than inclusion 1. The original compositions of the inclusions were likely similar and at least somewhat more iron-rich than they are now. We conclude that the two inclusions originated from an L chondrite, a result consistent with the element and oxygen-isotope data from the host chrome-spinel [4].

The STEM map also shows an exsolution pattern of relatively Ti-enriched chrome-spinel lamellae (A) and surrounding Fe-enriched chrome-spinel (B). The concentration of lamellae between the inclusions suggests that the formation of the inclusions strained the surrounding chrome-spinel. Without STEM maps, we would not have been able to observe the exsolution of chrome-spinel and gain insights into its formation.

A variety of microbeam techniques are employed to characterize and classify chrome-spinel grains from Jurassic sediments. Electron microprobe and ion microprobe analyses provide a robust classification system. Analyzing inclusions using the FIB and STEM also enhanced our ability to classify ambiguous grains, understand terrestrial alteration, and the formation of chrome-spinel [5].

\section{References:}

[1] B. Schmitz, Chem. der Erde 73 (2013), p. 117.

[2] C.E. Caplan et al, LPS XLIX (2017), Abstract \#2701.

[3] C. Alwmark and B. Schmitz, GCA 73 (2009), p. 1472.

[4] C.E. Caplan et al, LPS XLIX, (2017), Abstract \#2548.

[5] Supported by NASA grant NNX16AQ08G (GRH) and a European Research Council Grant (BS).
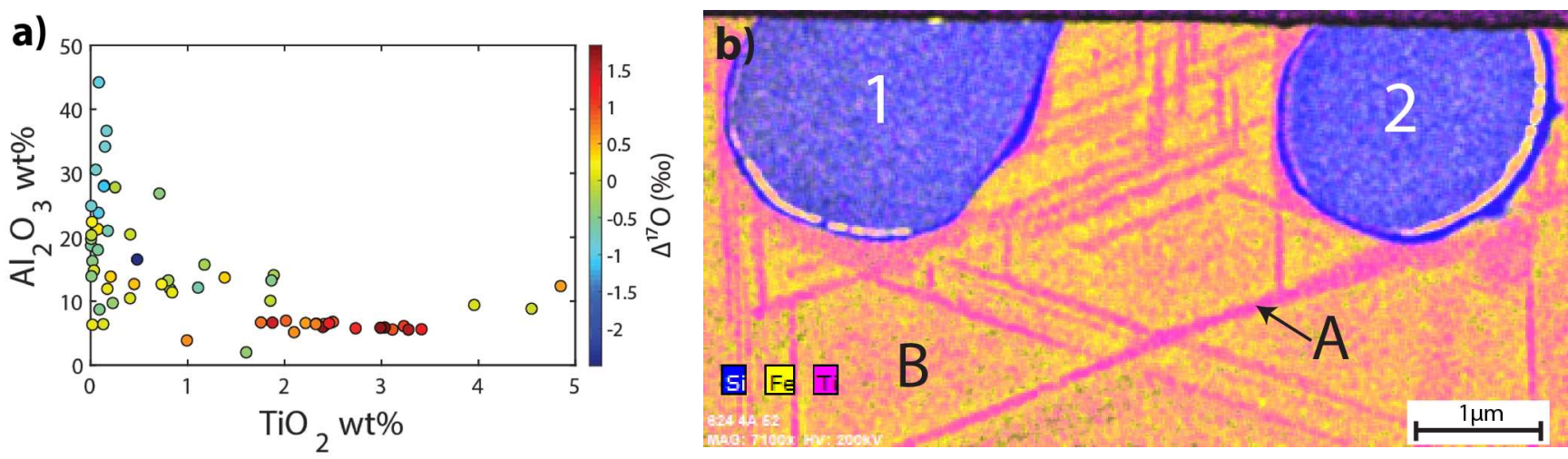

Figure 1. a) $\mathrm{Al}_{2} \mathrm{O}_{3}$ and $\mathrm{TiO}_{2}$ abundances for chrome-spinels. Oxygen isotope $\left(\Delta^{17} \mathrm{O}\right)$ color bar error is $\pm 0.36 \%$ o $(2 \sigma)[2]$. b) Overlaid STEM-EDS element maps of two pyroxene inclusions in chrome-spinel (blue $=\mathrm{Si}$, yellow $=\mathrm{Fe}$, magenta $=\mathrm{Ti}$ ) [4]. 